\title{
Lisa Anteby-Yemini, Juives et musulmanes. Genre et religion en négociation
}

Éditions Karthala, coll. « L'atelier méditerranéen », 2014, 252 p., Glossaire

\section{Sophie Nizard}

\section{(2) OpenEdition}

\section{Journals}

Édition électronique

URL : http://journals.openedition.org/assr/28280

DOI : $10.4000 /$ assr. 28280

ISSN : $1777-5825$

Éditeur

Éditions de l'EHESS

Édition imprimée

Date de publication : 31 décembre 2016

Pagination : 253

ISSN : 0335-5985

Référence électronique

Sophie Nizard, "Lisa Anteby-Yemini, Juives et musulmanes. Genre et religion en négociation », Archives de sciences sociales des religions [En ligne], 176 | octobre-décembre 2016, mis en ligne le 20 juillet 2017, consulté le 25 septembre 2020. URL : http://journals.openedition.org/assr/28280 ; DOI : https://doi.org/10.4000/assr.28280

Ce document a été généré automatiquement le 25 septembre 2020.

(c) Archives de sciences sociales des religions 


\section{Lisa Anteby-Yemini, Juives et musulmanes. Genre et religion en négociation}

Éditions Karthala, coll. « L'atelier méditerranéen », 2014, 252 p., Glossaire

Sophie Nizard

\section{RÉFÉRENCE}

Lisa Anteby-Yemini, Juives et musulmanes. Genre et religion en négociation, Éditions Karthala, coll. « L'atelier méditerranéen », 2014, 252 p., Glossaire.

1 Voici pour la première fois un ouvrage collectif qui adopte une approche résolument comparative de la question des femmes et plus généralement du genre dans l'islam et dans le judaïsme. Comme l'affirme Christian Bromberger dans sa préface, il s'agit d'une publication originale, car chaque contribution est écrite par deux chercheurs (soulignons que les auteurs sont en majorité des femmes), l'un spécialiste du judaïsme, l'autre de l'islam. Ces travaux font également la part des choses entre les normes religieuses (fixées dans les textes) et les pratiques sociales.

2 Ce comparatisme permet ainsi de dégager des similarités en matière de conception de la pureté des femmes, de mariage, d'accès des femmes aux textes et aux fonctions rituelles. En effet, le nombre important de prescriptions et d'interdits communs aux deux religions font dire à $\mathrm{C}$. Bromberger qu'on a affaire à des «traditions judéomusulmanes "; ce concept reste cependant à discuter et n'est d'ailleurs pas repris par les auteurs, d'autant que les deux traditions ne se sont pas nécessairement forgées dans les mêmes lieux. Mais, ce qui semble plus important encore est de dégager des différences mises en évidence dans la plupart des articles. On pourra regretter cependant que, très centrés sur le genre dans une perspective anthropologique, certains articles ne mettent pas suffisamment en avant les différences historiques et 
démographiques et appliquent de manière peu nuancée le terme de "contexte migratoire » aux deux groupes étudiés.

Dans son introduction, Lisa Anteby-Yemini explicite l'approche choisie : privilégier la notion de genre dans une analyse croisée des deux religions et comprendre "les stratégies de négociation et de contournement» utilisées par les femmes "afin de remettre en cause l'autorité masculine en matière de normes religieuses, de savoir juridique et de fonctions rituelles, ainsi que le contrôle des hommes sur leur corps et leur sexualité » notamment par le biais de revendications féminines, voire féministes, souvent similaires dans les deux religions. Elle insiste sur l'aspect « interreligieux » de ces articles écrits à quatre mains. Dans certains articles, en effet, il y a véritablement un effort de comparatisme et l'on perçoit une réflexion commune des deux auteurs, d'autres articles font plutôt l'effet d'une juxtaposition d'enquêtes déconnectées les unes des autres, ce qui donne une dimension artificielle à l'analyse commune.

4 L'ouvrage est centré sur trois thématiques : l'accès des femmes aux textes religieux, à l'espace du culte et aux fonctions rituelles (première partie), le mariage, la pureté, la sexualité et les stratégies de négociation correspondantes (deuxième partie) et la reproduction artificielle (procréation médicalement assistée ou PMA).

Dans son article d'ouverture, Nadine Weibel porte des regards croisés sur la régulation du féminin et tente d'éclairer le rapport des femmes aux religions en général. On assiste à partir des années 1960-70 à la multiplication des relectures des textes religieux mettant en avant des figures de femmes. Mais on constate aussi la résistance de certaines femmes elles-mêmes à toute évolution de leur place dans la religion et ceci dans les trois monothéismes; on est loin d'un consensus des croyantes sur la nécessité d'évolution de leur rôle. Malgré cela, remarque l'auteur, des changements sont en cours et donnent lieu à de nouveaux rituels (comme celui de la bat mitzva en milieu juif) ou à de nouvelles prières spécifiquement féminines. Le constat est également fait d'une réappropriation par les femmes de leur corps (voilement-dévoilement dans l'islam) et d'une vision plus égalitaire des responsabilités au sein du couple. Pour l'auteur, ces processus d'affirmation de soi sont une première étape vers l'autonomie des femmes et vers une religiosité féminine en devenir. Vision quelque peu optimiste, semble-t-il, du fait du retard et de la lenteur de ce processus au regard des avancées du féminisme en dehors du champ religieux.

6 L'accès des femmes juives et musulmanes à l'espace cultuel est saisi par l'article d'Annie Benveniste et de Marie-Laure Boursin ( Comment se construisent les espaces du culte dans les rapports de genre en islam et dans le judaïsme?») sur leur terrain respectif : Sarcelles et Marseille. Il s'agit pour elles de traiter ce sujet à partir de l'observation de "populations migrantes ». Les deux auteurs constatent une séparation des espaces et des rôles masculins/féminins dans les lieux de cultes juifs et musulmans, mais elles constatent aussi une séparation des rôles en fonction des degrés de savoir religieux et des générations (revendication, chez les plus jeunes femmes, d'un accès au savoir religieux en terrain juif par exemple). Les auteurs s'attachent enfin, d'un point de vue méthodologique, à la négociation de leur place d'enquêtrice sur leur terrain. À Sarcelles, en tant que juive, même non initiée, l'ethnologue est intégrée en vue de son éducation à la pratique souhaitée par les femmes rencontrées. En terrain musulman, la posture d'entre-deux de l'ethnologue (non musulmane) est la cause de son éviction, à l'initiative des femmes, de l'espace de la mosquée. 
7 Sonia Sarah Lipsyc et Belkacem Benzenine analysent dans leur article l'accès des femmes aux fonctions religieuses publiques dans le judaïsme et l'islam. Dans les deux contextes, les auteurs analysent les débats théologiques qui entourent ces changements.

8 Lipsyc retrace les étapes de l'accès des femmes à l'étude du Talmud. Elles occupent désormais de plus en plus souvent les fonctions d'avouées rabbiniques accompagnatrices des femmes dans leurs procédures de divorce religieux par exemple. Longtemps mises à l'écart de l'étude des textes juifs, les femmes commencent à y accéder (sous l'effet de leur accès aux études profanes et au monde du travail) à la fin $\mathrm{du} \mathrm{XIX}^{\mathrm{e}}$ siècle. $\mathrm{Au}$ cours $\mathrm{du} \mathrm{XX}^{\mathrm{e}}$ siècle, elles accèdent à l'étude du Talmud sous l'impulsion du Rabbin Soloveitchik, figure de proue du courant modern orthodox américain. L'auteur analyse l'influence de ces changements sur le judaïsme américain et plus tard israélien.

9 Benzenine fait le même constat pour l'accès des femmes aux fonctions d'imam, de muezzin, de mufti, de guide religieux qui révèlent des évolutions au sein de l'islam. En Islam (l'Algérie, le Maroc et l'Égypte sont pris comme terrains), l'accès à l'étude des textes est accepté pour mieux véhiculer l'islam auprès des femmes, avec un programme élaboré par les instances politiques religieuses dans le droit fil des tendances du "féminisme islamique » : relecture des textes sacrés par les femmes, notamment ceux relatifs à leur condition (inégalité entre les sexes, polygamie, droits des femmes) malgré l'opposition des mouvements conservateurs. Ces prérogatives (notamment celle de conseillère juridique) restent cependant toujours contrôlées par les autorités religieuses officielles des États. Des femmes sont aujourd'hui ordonnées rabbas aux États-Unis, mais en Israël, même si l'auteur constate une multiplication des femmes conseillères rabbiniques et rabbins ordonnées par des rabbins orthodoxes, elle relève les problèmes d'acceptation de ces femmes par une communauté. Une telle acceptation reste l'enjeu majeur de l'évolution du droit des femmes dans le judaïsme religieux. En Israël, il est courant, dans certaines synagogues égalitaires du courant modern orthodox, que des femmes participent à l'espace synagogal en faisant des sermons devant un public mixte et mènent une partie de l'office ou lisent publiquement la Torah. Certaines ont monté des groupes de prière spécifiquement féminins. D’autres femmes participent en Israël aux conseils municipaux religieux (malgré une forte opposition « sociétale »). La participation des femmes à la vie politique a suscité, à la naissance de l'État d'Israël, de fortes oppositions de la part de certains hauts dignitaires religieux. De la même manière, il y a eu en France de fortes tensions à propos de l'éligibilité des femmes aux Conseils d'administration des Consistoires. Pour l'auteur, il est nécessaire de réaffirmer à chaque époque ces droits. Cependant, remarque-t-elle, il n'y a toujours pas de femmes juges dans les tribunaux rabbiniques, cette situation étant particulièrement discriminatoire en Israël où les affaires de statut personnel (notamment les divorces) sont jugées devant ces juridictions.

Le Maroc a vu en 2005 la première promotion de femmes murchidat (guides religieuses ayant pour fonction la prédication, l'instruction religieuse, la visite des femmes en prison et diverses actions caritatives) bénéficiant d'une formation alliant sciences religieuses et sciences profanes et soutenues par le pouvoir royal dans sa politique de réforme, prévoyant plus de participation des femmes dans la société marocaine. Mais les autorités religieuses réaffirment régulièrement que les femmes ne peuvent être imams. On constate les mêmes processus en Algérie, toujours contrôlés par les 
instances religieuses du pays, mais contestés par les laïcs qui y voient une occasion d'islamisation de la société. En Égypte également, la formation de femmes murchidat est possible. Elles peuvent exercer la prédication, mais uniquement dans les mosquées sous l'autorité du Ministère des affaires religieuses et à condition de ne pas porter le voile intégral signe de radicalisation religieuse que l'état entend contrôler. D'autres questions sont discutées dans le droit islamique : est-il possible de créer des mosquées réservées aux femmes, est-il possible pour un homme de prier derrière une femme? C'est donc la question de la mixité des prières en islam qui est en débat.

11 Des résistances se font jour face à l'étude par les femmes du Talmud, notamment en France, et face à l'introduction de ces enseignements pour les filles dans les programmes scolaires des écoles juives. Ces résistances s'expliquent notamment par l'absence d'une orthodoxie moderne dans ce pays. En Israël, en dépit des avancées, il reste de fortes résistances de la part des orthodoxes, qui refusent la prise de parole publique des femmes et instrumentalisent la notion de pudeur pour justifier leur position. Les raisons de ces résistances (historiques, légales, sociologiques) sont longuement analysées par les auteurs; pourtant, Lypzic constate que la loi juive a su s'adapter, à certaines époques, aux changements intervenus dans les sociétés (interdiction de la polygamie au $\mathrm{XI}^{\mathrm{e}}$ siècle par un édit de Rabbenou Guershom, amélioration du statut des femmes grâce à des responsa rendues par des rabbins, tant ashkénazes que sépharades, etc.). L'une des principales raisons de ces résistances est le poids du conservatisme inhérent à toute structure religieuse et la confusion entre domination masculine et loi divine. Il faut y ajouter le refus de partager le pouvoir, la peur du changement liée à une peur du féminin ou à celle de perdre ses repères.

12 En islam, l'auteur soulève un autre type de négociation : des discussions ont lieu pour savoir si la fonction de mufti est accessible aux femmes. Cette fonction est inexistante en Algérie et au Maroc. Dans ce pays cependant, des femmes participent aux conseils collégiaux qui prononcent des fatwas. En Égypte, des femmes prononcent des fatwas uniquement au sujet des femmes. Quoi qu'il en soit, l'évolution du rôle des femmes vers le travail religieux (murchida) est en cours. Enfin, se font jour des formes modérées du discours politique sur les femmes, dans certains pays arabes ou musulmans, pour faire face à l'islamisme radical. Ceci a pour effet, selon Benzenine de diminuer la discrimination envers les femmes.

La deuxième partie de l'ouvrage s'ouvre sur l'article de Hanane Sekkat Hatimi et Emmanuela Trevisian Semi, consacré au mariage: "Les petites différences et les similarités : de la question de la dot et du contrat de mariage chez les juifs et les musulmans au Maroc ». Le nouveau code de la famille adopté au Maroc en 2004 est un acte législatif et social révolutionnaire du règne de Mohammed VI. Il instaure un contrat écrit qui représente une meilleure garantie pour les femmes sans déroger aux « lignes rouges » de la loi coranique imposées par les oulémas du royaume. Il révèle la volonté de garantir le droit des femmes à propos du divorce et de la polygamie, de préserver leur pension et leur patrimoine accumulé durant la vie commune. Le mariage est considéré par l'islam comme un acte religieux nécessitant un contrat de mariage : la kitba. Théoriquement, la fiancée peut exiger d'interdire au futur conjoint de prendre d'autres épouses et peut faire valoir son indisponibilité aux travaux ménagers... Mais de telles doléances sont rarement déclarées. Dans le passé, lors d'un mariage musulman, un tuteur représentait la fiancée. Les tuteurs avaient force de consentir et de conclure un mariage, même en l'absence de la femme. Le nouveau code n'exige plus 
l'intervention d'un tuteur et impose le consentement explicite de la fiancée ainsi que la mention de la dot versée par le futur mari au moment du mariage.

La ketoubbah est le contrat de mariage juif. Au Maroc, les mariages juifs étaient régis par deux codes différents en fonction de l'origine des mariés; le code de Castille réglait le mariage des Juifs d'origine espagnole (arrivés après l'expulsion des Juifs d'Espagne en 1492 - les megorachim), et interdisait la polygamie, le code des autochtones - appelé code biblique ou mosaïque - réglait les mariages des Juifs originaires du Maroc (tochavim). Dans le deuxième groupe, le mari pouvait s'engager à ne pas prendre une seconde épouse, sauf en cas de stérilité présumée de la première. Les Juifs du premier groupe étaient autorisés à divorcer en cas de stérilité. La bigamie pouvait également se justifier en cas de lévirat, institution qui oblige, en cas du décès d'un homme marié sans descendance, le frère du défunt soit à épouser la veuve soit à la libérer au cours d'une cérémonie appelée la halitsah. Si le frère était déjà marié, il pouvait donc avoir deux femmes. Certains rabbins ont exigé à partir du ${ }{ }^{e}{ }^{e}$ siècle qu'une femme donne son consentement en cas de volonté du mari d'épouser une deuxième femme.

Le douaire "fabuleux » de la femme juive (désigné dans l'article par le terme " dot ", employé par les musulmans) est vu par les femmes musulmanes marocaines comme la raison du nombre réduit de divorces dans les milieux juifs, car il décourageait les maris de répudier leur femme. Malgré l'obligation, dans le nouveau code de la famille, de stipuler le montant de la dot, les familles peuvent s'entendre pour faire figurer sur l'acte de mariage un montant symbolique, souvent pour faciliter le mariage. Mais les familles riches fixent des montants plus conséquents versés avant le mariage à la famille de la mariée pour préparer le mariage et la maison des futurs époux. L'article souligne la différence entre Juifs et musulmans sur le montant et la composition du douaire ainsi que sur le moment où il est payé à la femme. Mais il souligne surtout les similitudes entre les contrats de mariage juifs et musulmans : absence de signature de la femme au moment du mariage (ce qui a changé avec l'introduction du nouveau code marocain pour les femmes musulmanes); garantie de non-changement de résidence afin que la femme puisse rester proche de sa famille; existence d'une dot, qui peut être symbolique, et qui ne garantit pas nécessairement l'autonomie des femmes en cas de divorce.

16 Cet article nous fait prendre conscience du statut fortement inégalitaire des femmes dans les deux traditions religieuses, malgré les changements intervenus dans les dernières décennies. Dans le judaïsme comme dans l'islam, le divorce reste un acte unilatéral, certes encadré par la loi, mais qui soumet les femmes à la domination masculine en matière de droit de la famille.

17 Barbara Peveling et Simona Tersini consacrent quant à elles leur article aux questions de pureté chez les femmes juives et musulmanes «en contexte migratoire français». Au-delà d'un flou historique dans les parties de l'article consacrées à la pureté chez les femmes juives d'origine sépharade (l'exercice plus pointilleux des pratiques de purification rituelle en France est tantôt attribué à l'arrivée des Juifs d'Afrique du Nord en France, tantôt à l'action des groupes hassidiques plus orthodoxes déjà présents sur le sol français), cet article tente de repérer les enjeux des pratiques de purification féminines chez les Juives et les musulmanes de France.

Ce qui ressort de cette comparaison, au-delà d'une crispation commune sur la question de la pureté dans les deux groupes, ce sont les différences de raisons et d'enjeux des pratiques de purification. En contexte juif, la purification rituelle des femmes se 
rendant au bain rituel (miqvé) répond à des impératifs religieux normalisés liés à la vie conjugale: permettre les relations sexuelles par des rites de purification des femmes avant le mariage ou à l'issue d'une période d'interruption liée aux menstrues. Pour les musulmanes, les pratiques de purification (au hammam notamment) sont laissées à l'appréciation des femmes et à leurs représentations de ce qu'est une "bonne musulmane». Elles sont liées au besoin subjectif de purifier les corps pour pouvoir prier, cuisiner et participer à la vie religieuse collective.

Dans l'article de Martine Gross et Andrew Kam-Tuck Yip, il est question de l'homosexualité féminine dans le judaïsme et l'islam. Les auteurs soulignent la condamnation de l'homosexualité par l'ensemble des religions institutionnelles. C'est pourquoi ils reprennent dans un premier temps les textes religieux sur la question. Ils analysent ensuite la contestation en cours des discours dominants sur l'homosexualité et les positions socioculturelles des lesbiennes juives et musulmanes respectivement en France et en Grande-Bretagne.

20 Gross souligne l'invisibilité, dans les textes de la tradition juive, de l'homosexualité féminine; celle-ci n'est nulle part condamnée explicitement (contrairement à l'homosexualité masculine), ce qui ne revient pas à l'autoriser. Elle met en jeu deux dimensions d'interdits: la condamnation des pratiques homosexuelles et la transgression des rôles de genre. Suit une étude documentée de quelques commentaires du Talmud et avis ultérieurs faisant référence à l'homosexualité féminine pour la condamner, plus en tant que perte de contrôle d'un mari sur sa femme que comme pratique "abominable ». Cependant, la visibilité de ces pratiques et le fait qu'elles soient revendiquées aujourd'hui posent problème aux commentateurs contemporains "bien plus sévères que ceux du passé ", selon l'auteur. Au sein des courants contemporains du judaïsme, les réactions sont variées: alors que les plus libéraux bénissent les unions de même sexe et autorisent l'ordination de rabbins ouvertement gays ou lesbiennes (aux États-Unis notamment), les plus orthodoxes, reconnaissant depuis peu l'existence d'homosexuels dans leur rang, leur demandent généralement de s'abstenir. S'appuyant sur les textes islamiques (Coran, sharia et hadiths), les courants dominants de l'islam ne reconnaissent que l'hétérosexualité dans le mariage et considèrent l'homosexualité comme une révolte contre Allah et une violation de la nature. Le discours religieux dominant, hétéronormatif, est inextricablement lié au discours culturel sur les identités sexuelles et de genre, notamment à propos du mariage (seul lieu légitime pour la procréation). Dans la communauté musulmane britannique, l'homosexualité est considérée comme une "maladie occidentale» et les homosexuels musulmans comme "culturellement contaminés ». Les homosexuels sont donc soumis à des discriminations au sein et hors de leurs groupes religieux; les lesbiennes juives subissent de fait une triple discrimination du fait de leur triple statut minoritaire : en tant que Juives du fait d'une montée de l'antisémitisme partout constatée, en tant que femmes et lesbiennes elles font face à une discrimination liée à l'homophobie et au sexisme. A. Kamptuck Yip fait le même constat pour les lesbiennes musulmanes en Grande-Bretagne. Le racisme pouvant les rapprocher des communautés musulmanes, l'homophobie des milieux musulmans les en éloigner.

21 Face à ces discriminations, les auteurs constatent la mise en place de stratégies de résistance ; la plupart des femmes juives de l'enquête sont complètement à l'aise avec leur sexualité et les moins pratiquantes ne ressentent aucune incompatibilité entre leur 
orientation sexuelle et leur judéité. Les enquêtées se distancient généralement de l'autorité des rabbins, mais les plus pratiquantes cherchent à intégrer des synagogues accueillantes, marquant ainsi leur souhait de participer à la vie communautaire juive. Cependant, si certaines lesbiennes renoncent à fréquenter davantage la synagogue et même leur propre famille de peur d'être confrontées à un discours condamnant leur mode de vie, toutes les mères émettent le souhait de transmettre à leurs enfants le judaïsme (dans ses dimensions de mémoire, de traditions familiales et religieuses). Il en va de même pour les femmes souhaitant devenir mères. Ces enquêtes révèlent paradoxalement une fidélité des lesbiennes juives au judaïsme et à leur famille.

Aux États-Unis, des rabbins lesbiennes se penchent sur les textes de la tradition juive et les revisitent pour y rendre visible l'homosexualité féminine. Elles mettent en place des rituels de coming out devant la communauté, ce qui est rendu possible du fait de l'importance des courants libéraux dans ce pays.

En milieu musulman, de nombreuses lesbiennes s'efforcent d'obtenir le soutien de leur communauté malgré les stigmatisations qu'elles subissent, en affirmant une croyance inébranlable, mais aussi en réinterprétant les textes religieux par des stratégies de contestation de l'hétéronormativité et de l'homophobie, tendance désignée comme le queering des textes religieux. S'est ainsi développée une théologie gay et lesbienne musulmane sur le modèle des chrétiens homosexuels. Les homosexuels musulmans s'appuient également sur des réseaux associatifs de soutien qui permettent l'exploration par les lesbiennes musulmanes aussi bien de leur sexualité que de leur foi. La comparaison entre ces deux terrains permet aux auteurs de mettre en évidence des similarités (réinterprétation des textes, affirmation d'une sexualité positive, rejet des conceptions hétéronormatives, stratégies de résistance), mais aussi des différences quant aux enjeux de ces luttes : la plus forte politisation en milieu musulman, l'accent mis sur la transmission en milieu juif.

La troisième partie de l'ouvrage, consacrée à la reproduction artificielle, ne comprend qu'un seul article, celui de Liliane Vana et Sandra Houot intitulé « Regards croisés sur les défis de la PMA : loi juive (halakhah) et jurisprudence islamique (figh)». Cet article, à la fois très informatif et très analytique, est véritablement écrit à quatre mains et permet de prendre la mesure des enjeux éthiques et religieux que posent, au judaïsme et à l'islam sunnite, les avancées scientifiques en matière de procréation médicalement assistée et les situations inédites engendrées par ces nouvelles techniques.

Les deux traditions dissocient procréation et activité sexuelle: l'acte sexuel n'a pas pour seul objectif la procréation. Il doit répondre aux plaisirs respectifs des hommes et des femmes. Ainsi le plaisir sexuel est non seulement admis, mais valorisé dans la limite d'interdits propres à chaque loi. Si la procréation est dissociée de l'acte sexuel, elle est néanmoins un commandement, une mitzvah selon la loi juive, qui de manière inégalitaire ne s'impose qu'à l'homme. Ceci lui permet, en cas de stérilité de sa femme, de la répudier. Les autorisations de PMA en cas de stérilité de l'homme seront plus facilement accordées. Cependant en cas de PMA avec tiers donneur, l'homme ne sera pas considéré comme le père légal et n'aura pas accompli ce commandement. Dans l'islam, la procréation a pour finalité la filiation et fait partie des finalités de la loi, qui incombent tant aux hommes qu'aux femmes. Si le besoin d'enfant est pris en compte et considéré comme légitime par la loi islamique, le désarroi parental provoqué par l'incapacité de procréer ne permet cependant pas de recourir à la PMA considérée comme « embellissement et superflu». 

par des interprétations de la Loi permettent de contourner ces obstacles légaux posés a priori. L'insémination intraconjugale pose peu de problèmes aujourd'hui pour les décisionnaires religieux, mais la fécondation in vitro (FIV), même avec les gamètes des deux époux, est source de débats et de craintes, notamment du fait de l'indétermination des pouvoirs des médecins : certains décisionnaires juifs évoquent le risque que le sperme d'un étranger soit substitué à celui de l'époux, ce qui pourrait être assimilé à un adultère, d'autres autorisent la FIV avec les gamètes du mari à condition que le recueil du sperme et les manipulations se fassent par des personnes fiables et soucieuses de la loi juive. Du côté musulman, c'est la fécondation hors du corps féminin et ses conséquences éventuelles sur le fœtus qui posent question. l'acte médical en posant des limites. L'accès à un tiers donneur lors d'une fécondation médicale interpelle la halakhah comme le figh. Une telle fécondation est symboliquement chargée. Qualifiée d'immorale et donc interdite dans l'islam sunnite, elle est associée à un adultère pour certains décisionnaires juifs et donc porteuse de graves conséquences. Pourtant, l'une comme l'autre tradition tentent de trouver des accommodements pour la rendre possible.

Le Talmud envisage une conception sans rapport sexuel (à partir de sperme répandu dans un bain par exemple). La réception de sperme d'un autre homme que le mari et sans rapport sexuel n'est pas considérée comme un adultère, mais le père halakhique est le père biologique. Le risque à terme est l'inceste entre les enfants d'un même géniteur. Aussi, une PMA avec tiers donneur est possible, mais nécessite de connaître l'identité du donneur. Dans l'islam, la paternité légale prévaut. La question de l'inceste (du deuxième type) est abordée indirectement à partir du Coran et de l'histoire édifiante du prophète.

30 Les auteurs s'attachent enfin aux droits juif et musulman en matière de mères de substitution ou gestion pour autrui (GPA); dans les deux droits, la dissociation de la grossesse et de la maternité pose question. Qu'en est-il de la filiation ? Qui est la mère légale ? La génitrice, la gestatrice ou celle qui met l'enfant au monde ? À partir de quel stade l'embryon est-il considéré comme un être vivant doté d'une âme? De longs développements sont consacrés aux différents avis sur ces questions.

31 Finalement, le lecteur sort de cette lecture en ayant appris beaucoup sur la condition des femmes juives et musulmanes contemporaines, sur la manière dont elles négocient avec la loi religieuse et dont celle-ci s'impose ou non à elles, sur les droits juifs et musulmans concernant les femmes, leur pureté, leur sexualité, sur la capacité d'adaptation de ces droits religieux aux situations inédites que la modernité scientifique a engendrées et sur les débats entre décisionnaires religieux dans chacune des deux traditions religieuses. Mais cette lecture nous rend à la fois pleins d'optimisme devant les améliorations récentes du statut religieux des femmes mises en évidence par les auteurs et en même temps terriblement pessimistes tant le chemin à parcourir semble long avant d'arriver à une égalisation des conditions entre hommes et femmes dans ces deux grandes religions. On est en droit de se demander si cette égalisation est seulement possible au sein de certains courants du judaïsme et de l'islam. 